\title{
ISL1 expression is not restricted to pancreatic well-differentiated neuroendocrine neoplasms, but is also commonly found in well and poorly differentiated neuroendocrine neoplasms of extrapancreatic origin
}

\author{
Abbas Agaimy ${ }^{1}$, Katharina Erlenbach-Wünsch ${ }^{1}$, Björn Konukiewitz ${ }^{2}$, Anja M Schmitt ${ }^{3}$, \\ Ralf J Rieker ${ }^{1}$, Michael Vieth ${ }^{4}$, Franklin Kiesewetter ${ }^{5}$, Arndt Hartmann ${ }^{1}$, \\ Giuseppe Zamboni ${ }^{6}$, Aurel Perren ${ }^{3}$ and Günter Klöppel ${ }^{2}$ \\ ${ }^{1}$ Institute of Pathology, University Hospital, Erlangen, Germany; ${ }^{2}$ Department of Pathology, Technical \\ University of Munich, Munich, Germany; ${ }^{3}$ Department of Pathology, University of Bern, Bern, Switzerland; \\ ${ }^{4}$ Institute of Pathology, Klinikum Bayreuth, Bayreuth, Germany; ${ }^{5}$ Clinic of Dermatology, University Hospital, \\ Erlangen, Germany and ${ }^{6}$ Department of Pathology, Sacro Cuore Hospital of Negrar, Verona, Italy
}

\begin{abstract}
The human insulin gene enhancer-binding protein islet-1 (ISL1) is a transcription factor involved in the differentiation of the neuroendocrine pancreatic cells. Recent studies identified ISL1 as a marker for pancreatic well-differentiated neuroendocrine neoplasms. However, little is known about ISL1 expression in pancreatic poorly differentiated and in extrapancreatic well and poorly differentiated neuroendocrine neoplasms. We studied the immunohistochemical expression of ISL1 in 124 neuroendocrine neoplasms. Among pancreatic neuroendocrine neoplasms, $12 / 13$ with poor differentiation were negative, whereas $5 / 7$ with good differentiation but a Ki67 $>20 \%$ were positive. In extrapancreatic neuroendocrine neoplasms, strong positivity was found in Merkel cell carcinomas (25/25), pulmonary small cell neuroendocrine carcinomas (21/23), medullary thyroid carcinomas (9/9), paragangliomas/pheochromocytomas (6/6), adrenal neuroblastomas (8/8) and head and neck neuroendocrine carcinomas (4/5), whereas no or only weak staining was recorded in pulmonary carcinoids (3/15), olfactory neuroblastomas (1/4) and basaloid head and neck squamous cell carcinomas (0/15). ISL1 stained the neuroendocrine carcinoma component of 5/8 composite carcinomas and also normal neuroendocrine cells in the thyroid, adrenal medulla, stomach and colorectum. Poorly differentiated neuroendocrine neoplasms, regardless of their ISL1 expression, were usually TP53 positive. Our results show the almost ubiquitous expression of ISL1 in extrapancreatic poorly differentiated neuroendocrine neoplasms and neuroblastic malignancies and its common loss in pancreatic poorly differentiated neuroendocrine neoplasms. These findings modify the role of ISL1 as a marker for pancreatic neuroendocrine neoplasms and suggest that ISL1 has a broader involvement in differentiation and growth of neuroendocrine neoplasms than has so far been assumed.
\end{abstract}

Modern Pathology (2013) 26, 995-1003; doi:10.1038/modpathol.2013.40; published online 15 March 2013

Keywords: ISL1; ISL1-expression; neuroendocrine carcinomas; neuroendocrine neoplasms; neuroendocrine tumors; non-pancreatic tissues; pancreas

The human insulin gene enhancer-binding protein islet-1 (ISL1) is encoded by the $I S L-1$ gene. ${ }^{1}$ This transcription factor possesses two N-terminal LIM domains and one C-terminal homeodomain. ${ }^{2}$ LIM

Correspondence: Professor A Agaimy, MD, Pathologisches Institut, Universitätsklinikum Erlangen, Krankenhausstrasse 8-10, Erlangen 91054, Germany.

E-mail: abbas.agaimy@uk-erlangen.de

Received 5 October 2012; revised 18 December 2012; accepted 20

December 2012; published online 15 March 2013 domains (named after their discovery in the proteins Lin 11, ISL1 and Mec-3) are protein structural domains that mediate protein-protein interactions critical to cellular processes and have a role in the regulation of the cytoskeleton, organogenesis and oncogenesis. ${ }^{3}$ ISL1 has an important role in the embryogenesis and differentiation of the insulinproducing pancreatic beta cells within the islets of Langerhans. ${ }^{4-7}$ Deficiency of ISL1 was associated with absence of neural tube motor neuron 
differentiation in mouse experiments. ${ }^{8}$ Apart from its role in the cell line development of the endocrine pancreas, ISL1 was found to have a major role as a marker of cardiac progenitor cell lineage. ${ }^{9}$

Recently, ISL1 expression was studied in welldifferentiated pancreatic neuroendocrine neoplasms that are labeled by the WHO as neuroendocrine tumors (NETs). ${ }^{10,11}$ In these neoplasms, ISL1 proved to be a good marker for pancreatic NETs and their metastases, with a specificity of $78.4-100 \%$ and a sensitivity of $74.3-77.8 \% .^{10-12}$ Neuroendocrine carcinomas of pancreatic and extrapancreatic origin so far have not or only occasionally been tested for ISL1 expression. ${ }^{12-15}$ Following our own observation of a strong ISL1 reactivity in a case of a widely metastatic small cell neuroendocrine carcinoma of unknown origin, we launched this study with the aim to test the reliability of ISL1 as a marker for distinguishing pancreatic neuroendocrine carcinomas from their extrapancreatic counterparts and mimics. In addition, we also studied some well-differentiated extrapancreatic neuroendocrine neoplasms such as thyroid medullary carcinomas and paragangliomas/pheochromocytomas whose ISL1 status is not well known. In addition to ISL1, we stained a substantial fraction of the cases with TP53, which is known to be overexpressed in poorly differentiated neuroendocrine neoplasms of the pancreas ${ }^{16}$ and neuroendocrine carcinomas from extrapancreatic sites. ${ }^{17}$ We demonstrate in particular that ISL1 expression is mostly lost in pancreatic poorly differentiated neuroendocrine neoplasms, while it is almost ubiquitously observed in their extrapancreatic neuroendocrine counterparts. TP53 expression was inversely related to ISL1 positivity in poorly differentiated neuroendocrine neoplasms of the pancreas, but paralleled that of ISL1 in poorly differentiated extrapancreatic neuroendocrine neoplasms.

\section{Materials and methods}

A total of 124 neuroendocrine neoplasms of different origin (pancreas, gastrointestinal tract, skin, lung, thyroid, paraganglioma, adrenal glands, head and neck) and histological mimics such as head and neck basaloid squamous cell carcinomas $(n=15)$ were retrieved from the surgical pathology files of the Institutes of Pathology, Universities of Erlangen (Germany) and Bern (Switzerland) and the consultation files of three of the authors (AP, GZ and GK). Neuroendocrine neoplasms were diagnosed and classified according to the current World Health Organization (WHO) classification of NETs of the digestive tract. ${ }^{18,19}$ All tumors showed diffuse immunohistochemical expression of synaptophysin (antibody clone SY38, 1:50, Dako) with or without expression of chromogranin A (clone LK2H10, 1:500, Beckman-Coulter GmbH). In cases of Merkel cell carcinoma, neuroendocrine carcinomas of the head and neck region, small cell lung neuroendocrine carcinomas, pulmonary carcinoids, neuroblastomas, paragangliomas/pheochromocytomas, olfactory neuroblastomas, medullary thyroid carcinomas, and basaloid squamous cell carcinoma of head and neck the diagnosis was established where needed by using appropriate markers such as CK20, S100, calcitonin, CK5 and p63, respectively. The pancreatic neuroendocrine neoplasms were graded using the three-tiered grading system of the WHO that is based on the quantification of the nuclear labeling with Ki67 (MiB-1, 1:100, Dako). ${ }^{18-20}$ Welldifferentiated pancreatic neuroendocrine neoplasms G1 and G2 were called pancreatic NETs. Pancreatic neuroendocrine carcinomas were defined by their poor histological differentiation, with either small cell or large cell features. These G3-tumors show a Ki67 index above $20 \%$. For the purpose of this study, a subset of pancreatic neuroendocrine neoplasms that histologically looked like pancreatic NETs with trabecular patterns, but had a Ki67 index above $20 \%$, were ascribed to neuroendocrine neoplasms grade 3 to distinguish them from pancreatic neuroendocrine carcinomas of small or large cell types. Among the extrapancreatic malignancies there were also eight mixed neoplasms with a neuroendocrine component combined with other invasive or non-invasive neoplastic tissue.

Three $\mu \mathrm{m}$ thick sections were cut from one or two representative tumor tissue paraffin blocks and immunostained using a Ventana automated system (Ventana Medical Systems, Tucson, AZ, USA) and the following antibodies: ISL1 (clone 1H9, 1:1000, Abcam) and TP53 (clone DO-7, 1:50, Dako). Heatinduced antigen retrieval was performed for all antibodies using either protease 1 or cooking buffer CC1 (Ventana).

For ISL1, only nuclear staining was considered specific. Appropriate positive and negative controls were included. The immunohistochemical staining results were assessed using a four-tiered system: $0=$ negative $; 1+=<10 \% ; 2+=10-50 \% ; 3+=$ $>50 \%$. Tumors with scores $0 / 1+$ were considered negative and those scored $2+/ 3+$ were considered strongly positive. The intensity of staining was assessed as strong if similar or stronger than the staining of pancreatic islet cells, weak if barely discernible and moderate if in-between.

\section{Results}

The different entities of neuroendocrine neoplasms included in this study, and the immunohistochemical results for ISL1 are summarized in Table 1.

\section{Expression of ISL1 in WHO Grade 3 Pancreatic Neuroendocrine Neoplasms}

Thirteen cases of pancreatic neuroendocrine carcinomas of large $(n=11)$ and small cell type $(n=2)$ 
Table 1 Immunohistochemical expression of ISL1 in neuroendocrine neoplasms

\begin{tabular}{|c|c|c|c|c|c|c|}
\hline Tumor category & No. of cases & Score $3+$ & Score $2+$ & Score $1+$ & Score 0 & $\begin{array}{c}\text { Total } \\
(2+\text { and } 3+)(\%)\end{array}$ \\
\hline Pancreatic neuroendocrine carcinoma & 13 & 1 & 0 & $6^{\mathrm{a}}$ & 6 & $1(7.7)$ \\
\hline Pancreatic neuroendocrine neoplasm G3 & 7 & 3 & 2 & 0 & 2 & $5(71)$ \\
\hline Merkel cell carcinoma & 25 & 25 & 0 & 0 & 0 & $25(100)$ \\
\hline Small cell lung neuroendocrine carcinoma, primary & 13 & 13 & 0 & 0 & 0 & $13(100)$ \\
\hline Small cell lung neuroendocrine carcinoma, metastatic & 10 & 6 & 2 & 1 & 1 & $8(80)$ \\
\hline Small cell lung neuroendocrine carcinoma, total & 23 & 19 & 2 & 1 & 1 & $21(91)$ \\
\hline Large cell head and neck neuroendocrine carcinoma & 5 & 1 & 3 & 1 & 0 & $4(80)$ \\
\hline Medullary thyroid carcinoma & 9 & 8 & 1 & 0 & 0 & $9(100)$ \\
\hline Typical carcinoid lung & 10 & $1^{\mathrm{a}}$ & $1^{\mathrm{a}}$ & $1^{\mathrm{a}}$ & 7 & $2(20)$ \\
\hline Atypical carcinoid lung & 5 & 0 & $1^{\mathrm{a}}$ & 0 & 4 & $1(20)$ \\
\hline Neuroblastoma & 8 & 8 & 0 & 0 & 0 & $8(100)$ \\
\hline Paraganglioma/pheochromocytoma & 6 & 6 & 0 & 0 & 0 & $6(100)$ \\
\hline Olfactory neuroblastoma & 5 & 0 & 1 & 0 & 4 & $1(20)$ \\
\hline Mixed neoplasms ${ }^{\mathrm{b}}$ & 8 & 4 & 1 & 1 & 2 & $5(62)$ \\
\hline
\end{tabular}

${ }^{\mathrm{a}}$ Weak expression.

${ }^{\mathrm{b}}$ Carcinomas with components of poorly differentiated neuroendocrine neoplasm and non-neuroendocrine carcinoma.

Score definition: $0=$ negative; $1+=<10 \% ; 2+=10-50 \% ; 3+=>50 \%$.

and seven pancreatic neuroendocrine neoplasms grade 3 were studied. The latter tumors showed a well-differentiated trabecular-solid pattern that was in most parts indistinguishable from that of pancreatic NETs (Figure 1a) but exhibited a high mitotic activity and a Ki67 labeling above 20\% (Figure 1c). Of these seven neuroendocrine neoplasms grade 3, five were strongly positive for ISL1 (score $2+/ 3+$ ) (Figure 1b). All but 1 of the 13 neuroendocrine carcinomas scored negative $(0 / 1+)$ for ISL1 (Figures 1d-f). The six neuroendocrine carcinomas that scored only $1+$, showed a focal and weak expression. One neuroendocrine carcinoma of small cell type was diffusely positive, with moderate staining intensity. Comparison of ISL1 expression in poorly differentiated neuroendocrine carcinomas with that of TP53 revealed that all neuroendocrine carcinomas had a distinct TP53 expression in the range of $10-80 \%$ (mean, $48 \%$ ) that contrasted with the loss (or almost loss) of ISL1 expression (Figure 1f, inset). Four of the five neuroendocrine neoplasms grade 3 , in contrast had a very low TP53 expression $(<5 \%$; Figure 1c, inset), but showed strong and diffuse ISL1 reactivity. The one case of ISL1-negative neuroendocrine neoplasm grade 3 had a p53 index of $5 \%$. These results indicate an inverse correlation between the expression of TP53 and ISL1.

\section{Expression of ISL1 in Poorly Differentiated Extrapancreatic Neuroendocrine Neoplasms}

All 25 Merkel cell carcinomas showed a diffuse and strong $(3+)$ nuclear ISL1 staining (Figures2a and b). No difference was observed between primary tumors and metastases (data not shown). Isolated ISL1positive cells consistent with non-neoplastic Merkel cells were seen within the basal epidermis in 5 of 11 cases that contained normal skin next to the tumor (Figure 2c). All 13 primary and 8/10 metastatic pulmonary small cell neuroendocrine carcinomas expressed ISL1 strongly and diffusely (Figures 2d and e), whereas $2 / 10$ metastatic pulmonary small cell lung neuroendocrine carcinomas showed no or only focal and weak reactivity. Four of the five head and neck large cell neuroendocrine carcinomas from the oropharynx $(n=3)$ and the larynx $(n=2)$ showed a diffuse moderate expression, which was generally less intense than in Merkel cell carcinomas and small cell lung neuroendocrine carcinomas. The normal lung tissue next to small cell lung neuroendocrine carcinomas showed no ISL1 staining in the alveolar or bronchial epithelial cells. As in pancreatic neuroendocrine neoplasms, a subset of extrapancreatic neuroendocrine carcinomas was immunostained for TP53 and was found strongly positive ( $\geqslant 50 \%$ ) in $11 / 11$ small cell neuroendocrine carcinomas of the lung and 16/16 Merkel cell carcinomas, paralleling the intense expression of ISL1 (Figure 2b, inset and Figure 2f).

\section{Expression of ISL1 in Well-Differentiated Extrapancreatic Neuroendocrine Neoplasms}

All nine medullary thyroid carcinomas (Figure 3a) expressed diffusely ISL1, with a moderate nuclear staining intensity (Figure 3b). The thyroid tissue surrounding the carcinomas showed isolated ISL1positive cells that in consecutive sections were identified as calcitonin-positive parafollicular C cells (Figure 3c). Among the pulmonary carcinoids, only 3 of 15 cases (2/10 typical, 1/5 atypical) showed weak and scattered ISL1 positivity. Staining for TP53, which was performed in all medullary thyroid carcinomas and four pulmonary carcinoids, was negative or below $2 \%$ in all cases. 

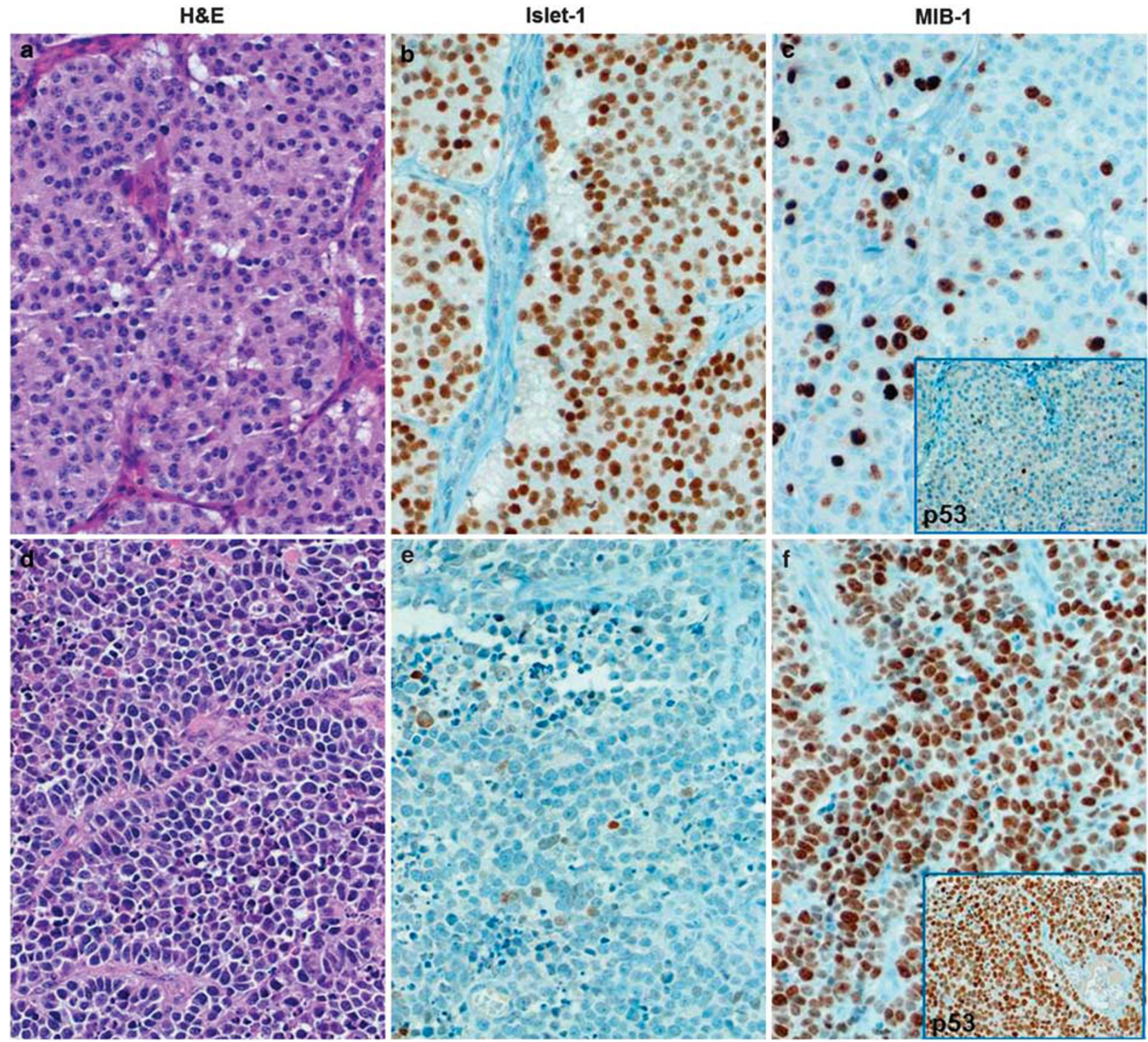

Figure 1 Grade 3 pancreatic neuroendocrine neoplasms with a trabecular-solid pattern (a) and a moderately intense, but diffuse ISL1 expression (b) and a high Ki67 index (c) but no staining for TP53 (c inset). In contrast, pancreatic small cell carcinoma (d) was almost negative for ISL1 (e). It displayed a very high Ki67 index (f) and TP53 (inset in f).

\section{Expression of ISL1 in Neuroblastic/Neuroectodermal} Tumors

All eight poorly differentiated (stroma-poor) adrenal neuroblastomas (Figure 4a) showed diffuse and strong nuclear staining, comparable to that seen in small cell lung neuroendocrine carcinomas and Merkel cell carcinomas (Figure 4b) with a similarly strong TP53 expression (Figure 4c). The six paragangliomas/pheochromocytomas tested were also diffusely positive, but stained less intensively than the neuroblastomas (Figures $4 \mathrm{~d}$ and e). Only one (grade 2 according to the WHO classification) ${ }^{21}$ of five olfactory neuroblastomas showed moderate staining, the remaining cases (all grades 3 or 4) were negative. All cases of neuroblastoma and pheochromocytoma showed moderate nuclear staining in the adjacent non-neoplastic cells of the adrenal medulla, with no staining in the cortex (Figure 4f).

\section{Expression of ISL1 in Gastroesophageal Neuroendocrine Carcinomas and in Composite and Non-Neuroendocrine Carcinomas}

This group of neoplasms included mixed adenoneuroendocrine carcinomas of the stomach/gastroesophageal junction $(n=2)$ and the rectum $(n=2)$, one tubulovillous adenoma of the rectum combined with a large cell neuroendocrine carcinoma, one basaloid squamous cell carcinoma of the tonsil combined with small cell neuroendocrine carcinoma, one adenosquamous carcinoma of the lung combined with small cell neuroendocrine carcinoma 

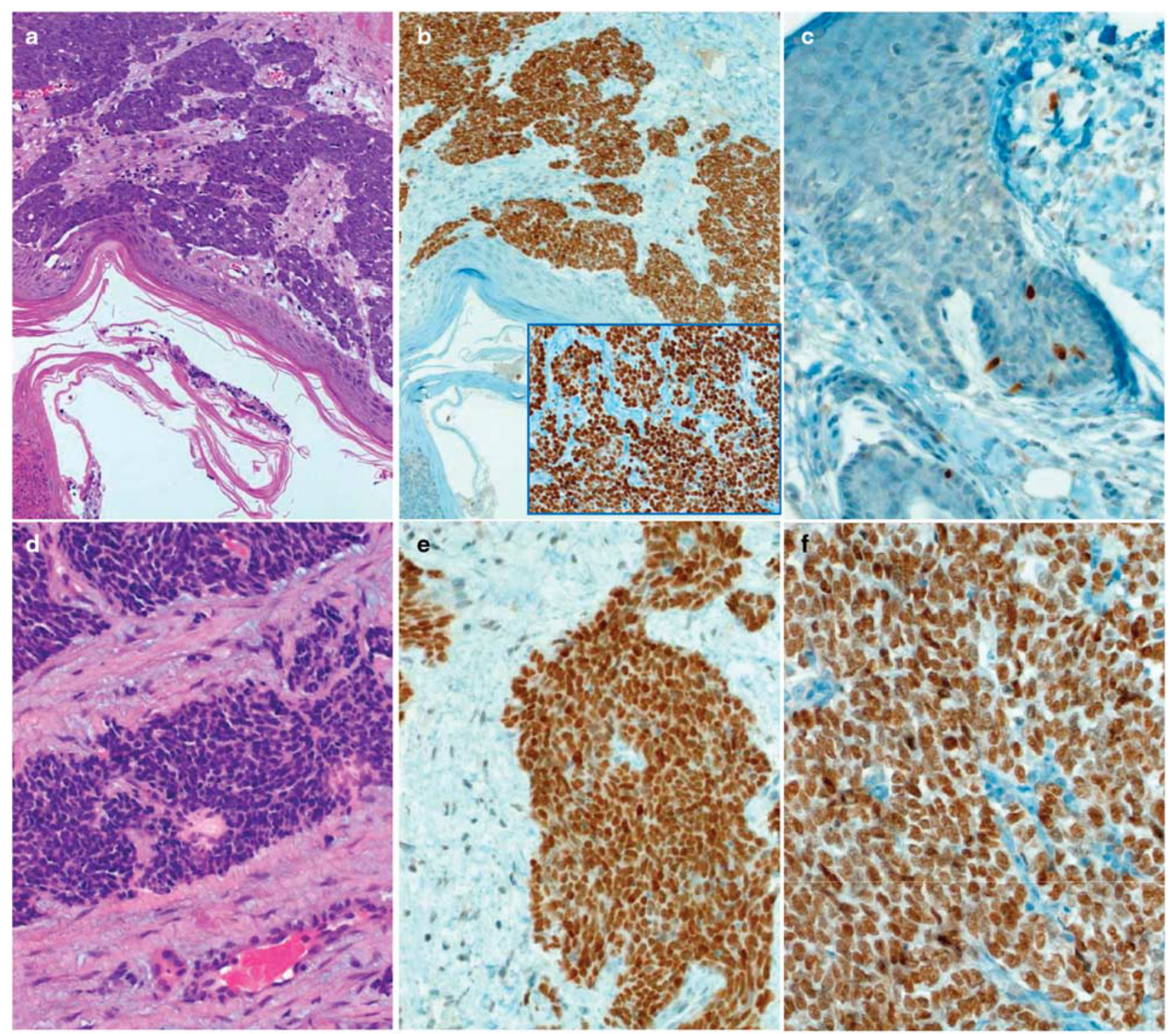

Figure 2 Merkel cell carcinoma of skin (a) with diffuse and intense positivity for ISL1 (b) and TP53 (b inset). Isolated ISL1-positive cells were seen within the basal epidermis consistent with normal Merkel cells (c). Small cell lung neuroendocrine carcinoma metastatic to the liver (d) showed diffuse expression of ISL1 (e) and TP53 (f).

and one squamous cell carcinoma of the skin combined with a Merkel cell carcinoma. Strong and diffuse $(2+/ 3+)$ ISL1 expression was seen in five of these cases, involving only the neuroendocrine carcinoma component and completely sparing the non-neuroendocrine areas. Among the three rectal cases, one mixed adenoneuroendocrine carcinoma and the composite adenoma neuroendocrine carcinoma were completely negative, whereas the second mixed adenoneuroendocrine carcinoma showed strong expression in the neuroendocrine carcinoma area. The gastric mixed adenoneuroendocrine carcinoma showed diffuse and strong expression associated with diffuse TP53 positivity in the neuroendocrine carcinoma component. The gastroesophageal junction tumor was negative for ISL1 and showed TP53 expression in $>60 \%$ cells. In the rectal and gastroesophageal specimens, isolated strongly staining cells were seen within the neighboring basal mucosal crypts, consistent with neuroendocrine cells. All other composite carcinomas (skin, tonsil and lung) showed strong diffuse staining limited to the neuroendocrine carcinoma component. The 15 basaloid squamous cell carcinomas of the head and neck region, which histologically very much resembled poorly differentiated neuroendocrine neoplasms, were all negative for ISL1 staining.

\section{Discussion}

ISL1 has been found to be a highly specific marker for pancreatic NETs that are by definition well-differentiated neuroendocrine neoplasms. ${ }^{10-12}$ This study, which in its first part focused on pancreatic neuroendocrine carcinomas, revealed 

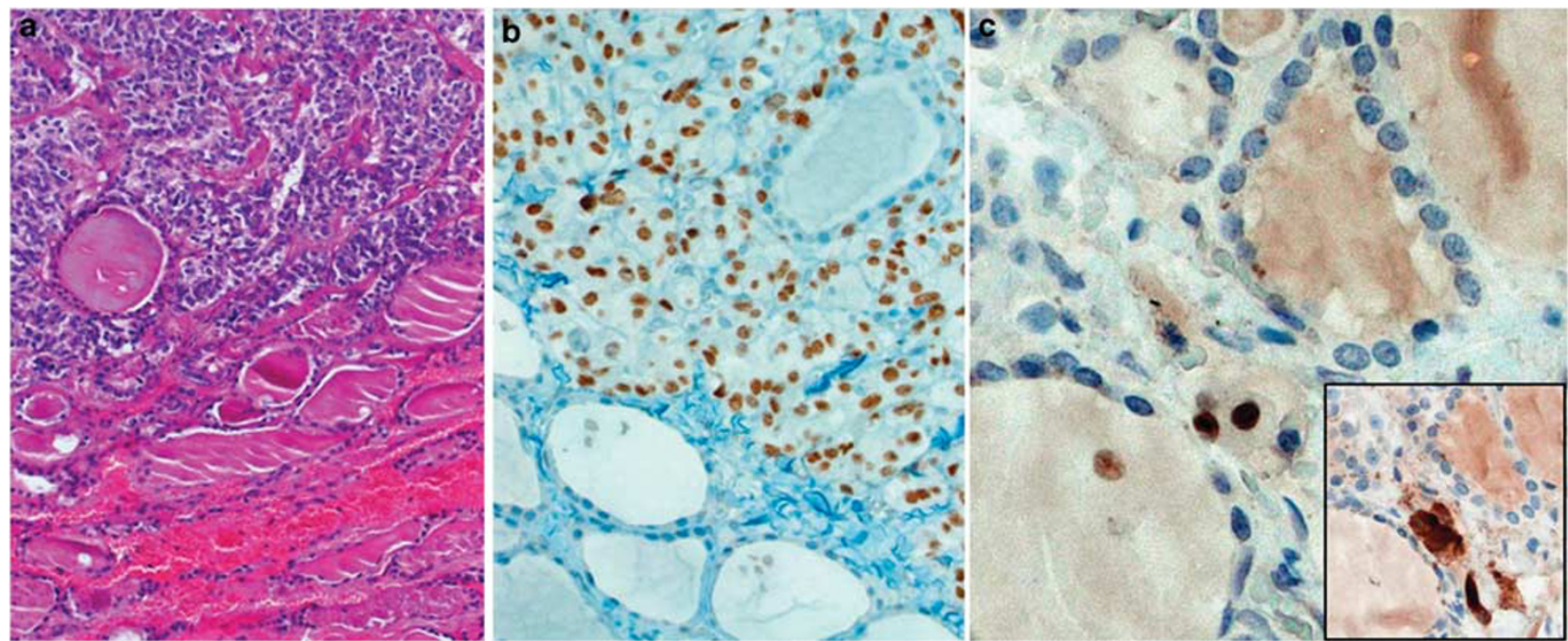

Figure 3 Medullary thyroid carcinoma (a, top) showed moderate to strong ISL1 expression (b). Isolated C cells distant from the tumor were immunoreactive for ISL1 (c main image) and calcitonin (c inset, serial sections).

that ISL1 is not a loco-specific marker for these poorly differentiated neuroendocrine neoplasms, because they are either negative or only very weakly and focally positive for ISL1. The data of the study's second part demonstrated, on the other hand, an almost ubiquitous expression of ISL1 in certain extrapancreatic neuroendocrine neoplasms, with the highest labeling detected in neuroendocrine carcinomas of small and round cell morphology (Merkel cell carcinomas, small cell lung neuroendocrine carcinomas and adrenal neuroblastomas) followed by an intermediate expression in paragangliomas/pheochromocytomas, medullary thyroid carcinomas and gastrointestinal neuroendocrine carcinomas. Only weak or absent reactivity was observed in pulmonary carcinoids, olfactory neuroblastomas and all non-NET tissues tested.

The results show that expression of ISL1 is related to certain groups of neuroendocrine neoplasms and is absent from the non-NETs so far tested. In the pancreas, where ISL1 is expressed in the normal islet cells, it is known to represent a differentiation marker. ${ }^{4-8}$ Thus, expression of ISL1 in pancreatic NETs most likely reflects the high differentiation status of these neoplasms. ${ }^{10,11}$ Accordingly, it is not surprising that ISL1 is lacking or only weakly expressed in poorly differentiated pancreatic neuroendocrine carcinomas. Interestingly, ISL1 expression was preserved in a subset of pancreatic neuroendocrine neoplasms, which were assigned grade 3 because of their high proliferation index (Ki67>20\%), ${ }^{18-20}$ but still retained the typical histological pattern of NETs, thus clearly deviating in their histological differentiation from poorly differentiated neuroendocrine neoplasms of small or large cell type. This observation supports our interpretation of a differentiation-associated expression of ISL1 in pancreatic neuroendocrine neoplasms and may also serve as a further argument that some pancreatic grade 3 neuroendocrine neoplasms, although overlapping in their proliferative activity with poorly differentiated neuroendocrine carcinomas, still remain distinct from the latter neoplasms.

Another finding for the notion that pancreatic NETs and neuroendocrine carcinomas are fundamentally distinct neoplasms, probably differing in their genetic structure, is the inverse correlation of ISL1 and TP53 positivity in this study. In a recent study, pancreatic NETs revealed an absent or only very low TP53 signature in the presence of high expression of PDX1, whereas pancreatic neuroendocrine carcinomas, regardless whether of small or large cell type, showed the opposite. ${ }^{16}$ Our results are consistent with these data. These features have also been observed in large cell neuroendocrine carcinomas and atypical carcinoids of the head and neck, and have been used as a distinguishing criterion. ${ }^{17,21}$

In extrapancreatic neuroendocrine neoplasms, including neuroendocrine neoplasms of neuroblastic origin, ISL1 expression lacks the relationship to histological differentiation seen in pancreatic neuroendocrine neoplasms. Almost all extrapancreatic neuroendocrine carcinomas that were studied including Merkel cell carcinomas and neuroendocrine carcinomas of the lung and head and neck region strongly expressed ISL1. This pattern was also found in all stroma-poor neuroblastomas and many of the small series of neuroendocrine carcinomas and mixed adenoneuroendocrine carcinomas in the gastrointestinal tract. Interestingly, the TP53 expression in these poorly differentiated extrapancreatic neuroendocrine neoplasms positively correlated with that of ISL1, thus contrasting sharply with the situation in pancreatic neuroendocrine neoplasms.

Among the extrapancreatic neuroendocrine neoplasms that we considered well-differentiated, the 


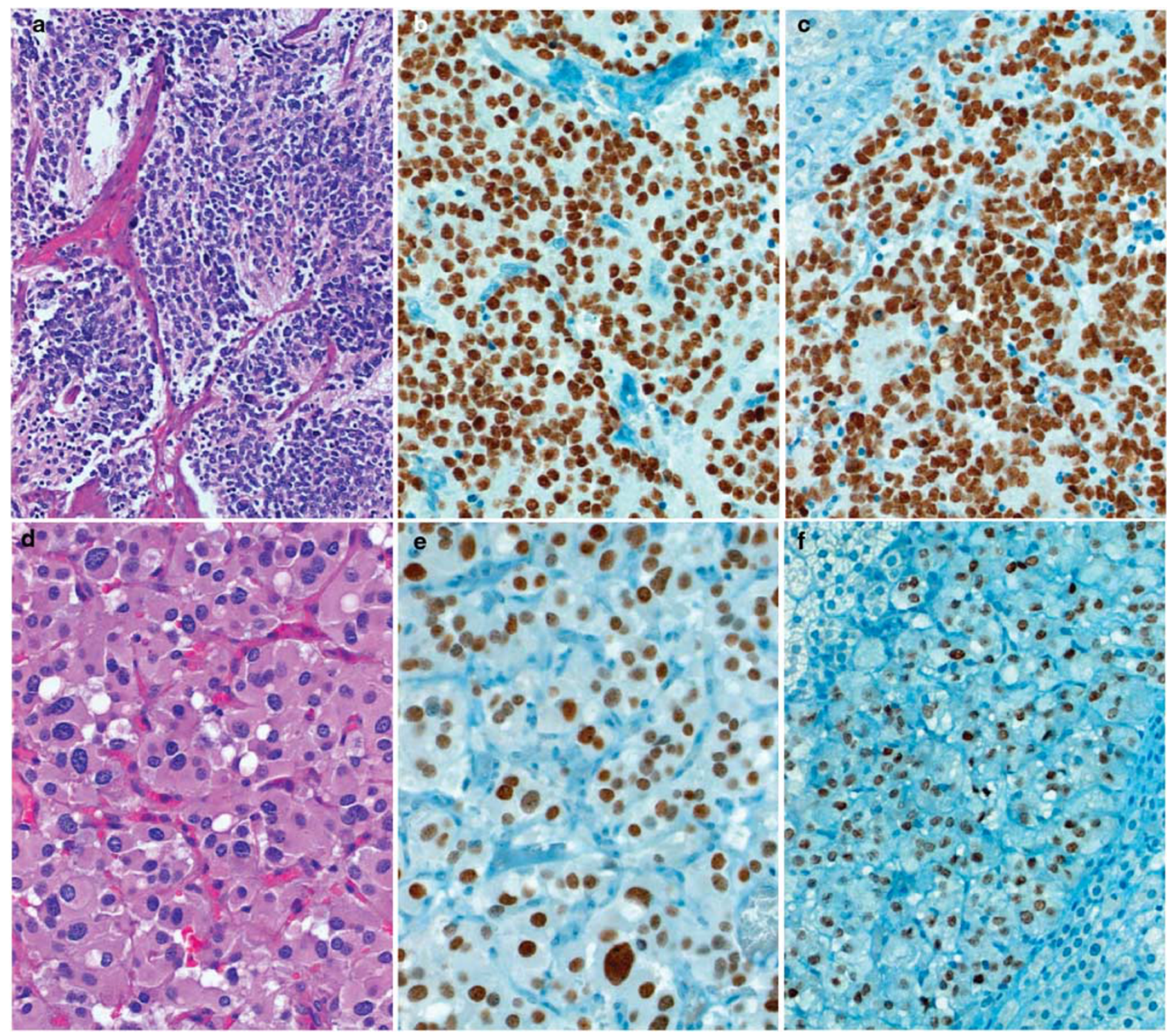

Figure 4 Adrenal neuroblastoma with characteristic large nests of small round cells (a) strongly expressed ISL1 (b) and TP53 (c). Carotid body paraganglioma (d) revealed moderate diffuse reactivity with ISL1 (e). (f) Normal adrenal medulla expressed ISL1 with a moderate intensity. Note the non-staining adrenal cortical cells (upper left and lower right field) surrounding the medulla.

medullary thyroid carcinomas and paragangliomas/ pheochromocytomas stained strongly for ISL1, whereas typical and atypical pulmonary carcinoids and olfactory neuroblastomas did not. To explain this difference, we followed the assumption that the identification of ISL1-positive cells in non-neoplastic cells next to the tumors could suggest, like in the pancreas, a role for ISL1 as differentiation factor in these tissues. We observed no ISL1 expression in the normal lung and sinonasal mucosa, explaining its absence in their respective tumors, but detected single positive cells in the thyroid and adrenal medulla, consistent with thyroid $\mathrm{C}$ cells and normal adrenal medullary cells. It is therefore possible that ISL1 expression in medullary thyroid carcinomas, like in pancreatic NETs, reflects the high degree of differentiation of these tumors. This notion is also supported by the lack of abnormal expression of
TP53. However, the finding that ISL1 expression is even stronger in the poorly differentiated neuroblastoma than in the paragangliomas/pheochromocytomas is not consistent with the above interpretation and leaves the role of ISL1 in the development of these tumors unclear.

This is also the case in neuroendocrine carcinomas of the gastrointestinal tract, whether occurring as pure neuroendocrine carcinoma or as a neuroendocrine carcinoma component in mixed adenoneuroendocrine carcinoma. These tumors showed a heterogeneous expression, with some cases being completely negative and others strongly positive. The expression of ISL1 in normal endocrine cells of the stomach and colorectum contrasts with its complete loss in some gastrointestinal neuroendocrine carcinomas, similar to their pancreatic counterparts. Although the number of cases included in 
this study is too low to allow for conclusive results, it is possible that the group of neuroendocrine carcinomas of the GI tract is heterogeneous, depending on factors such as location and pathogenesis.

From a differential diagnostic point of view, our data are important in highlighting for the first time the ubiquitous expression of ISL1 in common extrapancreatic poorly differentiated neuroendocrine neoplasms such as small cell neuroendocrine carcinoma of the lung and Merkel cell carcinomas, but also in some well-differentiated neuroendocrine neoplasms such as medullary thyroid carcinoma or paraganglioma/pheochromocytoma. These findings have to be especially kept in mind when dealing with liver metastases of unknown primaries. Thus, in case of an ISL1-positive liver metastasis of a welldifferentiated neuroendocrine neoplasm, the primary will most likely be found in the pancreas, but in rare instances may be located in the thyroid (medullary carcinoma) or the adrenal gland (pheochromocytoma). For these rare cases, the use of a well-selected immunohistochemical marker panel (cytokeratin, calcitonin, CEA and TTF-1) seems to be necessary for an appropriate differential diagnostic workup. On the other hand, in case of a ISL1positive poorly differentiated neuroendocrine neoplasm metastatic to the liver, different locations have to be considered as site of the primary such as the lung, skin, head and neck, or gastrointestinal tract. For these neoplasms, ISL1 is only of little help in indicating the site of the primary.

In summary, the transcription factor ISL1 is expressed in well differentiated but barely in poorly differentiated pancreatic neuroendocrine neoplasms. Furthermore, it is commonly found in diverse extrapancreatic poorly differentiated neuroendocrine neoplasms and in a subset of welldifferentiated extrapancreatic neuroendocrine neoplasms such as medullary thyroid carcinoma and paraganglioma/pheochromocytoma. These heterogeneous findings point to different roles ISL1 is having in the development of pancreatic and non-pancreatic neuroendocrine neoplasms, probably depending on tumor type and origin.

\section{Acknowledgements}

We are grateful to Claudia Störer (immunohistochemistry laboratory, Erlangen) for excellent technical assistance. We also thank Dr Anne Hoorens, Department of Pathology, VUB Brussels, Belgium, for contributing a case.

\section{Disclosure/conflict of interest}

The authors declare no conflict of interest.

\section{References}

1 Karlsson $\mathrm{O}$, Thor $\mathrm{S}$, Norberg $\mathrm{T}$, et al. Insulin gene enhancer binding protein Isl-1 is a member of a novel class of proteins containing both a homeo- and a Cys-His domain. Nature 1990;344:879-882.

2 Wang M, Drucker DJ. The LIM domain homeobox gene isl-1: conservation of human, hamster, and rat complementary deoxyribonucleic acid sequences and expression in cell types of nonneuroendocrine lineage. Endocrinology 1994;134:1416-1422.

3 Zheng Q, Zhao Y. The diverse biofunctions of LIM domain proteins: determined by subcellular localization and protein-protein interaction. Biol Cell 2007;99:489-502.

4 Ahlgren U, Pfaff SL, Jessell TM, et al. Independent requirement for ISL1 in formation of pancreatic mesenchyme and islet cells. Nature 1997;385: 257-260.

5 Hori Y, Gu X, Xie X, et al. Differentiation of insulinproducing cells from human neural progenitor cells. PLoS Med 2005;2:e103.

6 Guo $\mathrm{T}$, Wang W, Zhang $\mathrm{H}$, et al. ISL1 promotes pancreatic islet cell proliferation. PLoS One 2011;6:e22387.

7 Du A, Hunter CS, Murray J, et al. Islet-1 is required for the maturation, proliferation, and survival of the endocrine pancreas. Diabetes 2009;58:2059-2069.

8 May CL. The role of Islet-1 in the endocrine pancreas: lessons from pancreas specific Islet-1 deficient mice. Islets 2010;2:121-123.

9 Cai CL, Liang X, Shi Y, et al. Isl1 identifies a cardiac progenitor population that proliferates prior to differentiation and contributes a majority of cells to the heart. Dev Cell 2003;5:877-889.

10 Schmitt AM, Riniker F, Anlauf M, et al. Islet 1 (Isl1) expression is a reliable marker for pancreatic endocrine tumors and their metastases. Am J Surg Pathol 2008;32:420-425.

11 Hermann G, Konukiewitz B, Schmitt A, et al. Hormonally defined pancreatic and duodenal neuroendocrine tumors differ in their transcription factor signatures: expression of ISL1, PDX1, NGN3, and CDX2. Virchows Arch 2011;459:147-154.

12 Koo J, Mertens RB, Mirocha JM, et al. Value of Islet 1 and PAX8 in identifying metastatic neuroendocrine tumors of pancreatic origin. Mod Pathol 2012;25: 893-901.

13 Thor S, Ericson J, Brännström T, et al. The homeodomain LIM protein Isl-1 is expressed in subsets of neurons and endocrine cells in the adult rat. Neuron 1991;7:881-889.

14 Dong J, Asa SL, Drucker DJ. Islet cell and extrapancreatic expression of the LIM domain homeobox gene isl-1. Mol Endocrinol 1991;5:1633-1641.

15 Buxbaum P, Horvat G, Gamper C, et al. Monoclonal islet antibody HISL-19 as a tool in the diagnosis of neuroendocrine carcinomas of the skin. Cancer Detect Prev Suppl 1987;1:263-268.

16 Yachida S, Vakiani E, White CM, et al. Small cell and large cell neuroendocrine carcinomas of the pancreas are genetically similar and distinct from well-differentiated pancreatic neuroendocrine tumors. Am J Surg Pathol 2012;36:173-184.

17 Kao HL, Chang WC, Li WY, et al. Head and neck large cell neuroendocrine carcinoma should be separated from atypical carcinoid on the basis of different 
clinical features, overall survival, and pathogenesis. Am J Surg Pathol 2012;36:185-192.

18 Klimstra DS, Arnold R, Capella C, et al. NENs of the pancreas, In: Bosman FT, Carneiro F, Hruban RH, Theise ND (eds). World Health Organization Classification of Tumours of the Digestive System. IARC: Lyon, France; 2010, pp 322-326.

19 Klöppel G. Classification and pathology of gastroenteropancreatic neuroendocrine neoplasms. Endocr Relat Cancer 2011;18(Suppl 1):S1-16.
20 Yang Z, Tang LH, Klimstra DS. Effect of tumor heterogeneity on the assessment of Ki67 labeling index in well-differentiated neuroendocrine tumors metastatic to the liver: implications for prognostic stratification. Am J Surg Pathol 2011;35: 853-860.

21 Barnes L, Eveson JW, Reichart P, Sidransky D (eds). World Health Organization Classification of Tumours. Pathology and Genetics of Head and Neck Tumours. IARC Press: Lyon, France, 2005;p275. 